\title{
Glucose-6-phosphate dehydrogenase (G6PD) activity of human sperm
}

\author{
SIDDHARTHA SARKAR, ALVIN J. NELSON, AND O. W. JONES
}

From the Division of Medical Genetics, Department of Medicine (M-013), University of California, San Diego, La Jolla, California, USA

SUMMARY Sperm from normal human donors and a G6PD-A- individual were examined for $\mathrm{X}^{\mathrm{\omega}}$ linked glucose-6-phosphate dehydrogenase activity and autosomally linked 6-phosphogluconate dehydrogenase activity. With the use of fluorescence microscopy, we devised a procedure to visualise in individual sperm cells the fluorescence of reduced coenzyme NADPH formed by each of the two enzymes in the presence of appropriate substrates. We found significant differences in the populationi distribution of sperm expressing each of the two activities, and the ratio of the two activities in spermp homogenate is very different from the one found in erythrocyte lysates. The possibility of haploid gene expression has been considered in interpreting these results.

Because of asymmetrical segregation of the sex chromosomes in male meiosis, it is of particular interest to know if the expression of the $\mathrm{X}$-linked markers is limited to those sperm, about a half of the total numbers, that carry and can transmit the X-chromosome. In initial studies with human sperm we detected the activities of 4 X-linked enzymes (McKusick, 1975a): alphagalactosidase, phosphoglyceratekinase, hypoxanthineguanine phosphoribosyltransferase, and glucose-6-phosphate dehydrogenase. G6PD activity appeared to be ideally suitable among the 4 enzymes for detecting sexlinked expression of individual sperm cells. This brief report presents the evidence to indicate that the sperm G6PD is probably identical to the erythrocyte enzyme (Yoshida, 1966) and a single locus in the X-chromosome controls the expression of G6PD in sperm as in other tissues (Linder and Gartler, 1965). With the use of fluorescence microscopy, we devised a procedure to visualise, in individual sperm cells, the formation of reduced coenzyme (NADPH). Using this procedure we have determined the population distribution characteristic of G6PD, and autosomally linked 6PGD (6-phosphogluconate dehydrogenase) activities in normal sperm (phenotype G6PD-B ${ }^{+}$) and sperm from a G6PD-deficient donor (phenotype G6PD-A-) (Yoshida et al., 1967).

\section{Materials and methods}

ENZYME ASSAY

A fluorimetric method (Estabrook and Maitra, 1962) was used to assay the production of NADPH Received for publication 18 October 1976 (nicotinamide adenine dinucleotide phosphate, re duced) from NADP in the presence of glucose- $6 \overrightarrow{-}$ phosphate (G6P) or 6-phosphogluconate (6PG) Enzyme activities were measured in a Farrand spectrofluorometer with 2 grating monochromators one to select the wavelength of excitation $(365 \mathrm{~nm}$ for reduced NADPH and the other (set at $472 \mathrm{~nm}$ ) to receive the emitted wavelengths. In addition, the्ల emitted wavelengths were passed through a sharm cut-off filter excluding the excitation wavelengths $\overrightarrow{\vec{\sigma}}$ Continuous recording of NADPH level was made in Honeywell model Electronik 19; the scale was cali? brated by a solution of NADPH and the yeast enzyme. (Zwischenferment) was used as a standard to nor malize different assays. Assay mixtures containe either $1 \mathrm{~mm}$ glucose-6-phosphate (G6P) or 6-phos음 phogluconate (6PG), $70 \mu \mathrm{M}$ NADP, $7 \mathrm{~mm}$ magnesium chloride, in $50 \mathrm{~mm}$ Tris buffer, $\mathrm{pH} \mathrm{8.0.} \mathrm{The} \mathrm{ex仓}$ traction of enzymes from sperm required the use of $0.5 \%$ noniodet $\mathrm{P}-40$ in the homogenate. The de? tergent was also added to the erythrocyte lysate to maintain the same assay conditions for both extracts을. Three other additives were required to stabilisథ enzyme activity in stored extracts, $10 \mu \mathrm{M}$ NADP $1 \mathrm{~mm} \beta$-mercaptoethanol, and $1 \mathrm{mg} / 100 \mathrm{ml}$ soybear trypsin inhibitor. One million intact sperm cells or an equivalent amount of extract were used for routine assays of enzyme activity.

FLUORESCENCE MICROSCOPY OF NADPH FORMATION

The fluorimetric principle in measuring NADPH formation was applied in fluorescence microscopy t $\vec{\Phi}$ visualize G6PD or 6PGD activity in individuad 
sperm cells. Dark field illumination was achieved by the use of an ultracondenser in a Zeiss microscope, Lamp HBO 200, excitation filter BG and barrier filter 44. Sperm suspension was made from the pellet derived after 2 centrifugations of fresh semen on a layer of $15 \%$ sucrose in $0.15 \mathrm{M}$ sodium chloride solution containing $10 \mu \mathrm{M}$ NADP and $1 \mathrm{mmol}$ $\beta$-mercaptoethanol. Contamination level of cells other than sperm was in the range of 0.5 to $5 \%$. Sperm was finally resuspended in the assay mixture. A wet preparation was mounted immediately on a slide (no. 7038, Arthur Thomas) with a no. 1 coverglass. The coverglass was sealed and the slide was incubated at room temperature for $\mathbf{3 0}$ minutes before exposing for photomicrography (Tri-X Pan, Kodak, ASA 400, black and white film; exposure time 1 to 2 minutes).

STAINING OF ENZYME BANDS IN ACRYLAMIDE GEL AND DISC GEL ELECTROPHORESIS

This was carried out as described previously (Howell et al., 1972).

\section{NEUTRALIZATION OF ENZYME ACTIVITY BY} RABBIT ANTIBODY

The fluorimetric technique was used to assay the neutralizing activity of the rabbit antibody. The inhibition of enzyme activity by the rabbit antibody was almost immediate. This was titrated directly in the fluorimeter by the decrease in the slope of the enzyme reaction following the addition of antibody; under these conditions, approximately 0.025 units of G6PD activity ( 1 unit $=1 \mu \mathrm{mol}$ NADPH formed per minute at 25 degrees) was neutralized by $5 \mu \mathrm{l}$ undiluted antibody. The rabbit antibody was a gift from Dr A. Yoshida (Yoshida et al., 1967).

\section{Results and discussion}

The recovery of G6PD activity in sperm homogenates is highest when extracts are prepared in the presence of a nonionic detergent, such as noniodet P-40. It may be that the native enzyme is in the particulate fraction, or has a tendency to bind to lipids released during homogenization. Given the low water content and high lipid content of sperm it is not surprising that the enzyme is found in an insoluble form, and can be solubilised by extracting with a mild detergent. The average G6PD activity of sperm is $0.002 \mu \mathrm{mol}$ NADPH produced per minute per million cells or equivalent extract, compared with 5 times that activity in erythrocyte lysates. However, G6PD of the sperm extracts and erythrocyte lysates migrate to an identical position in disc gel electrophoresis: the single band of G6PD activity, revealed by specific staining of the acrylamide gel after disc electro-

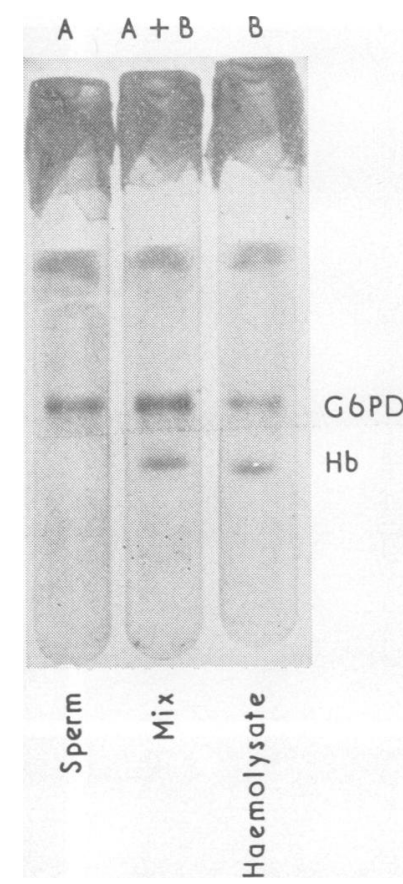

Fig. 1 Acrylamide gel electrophoregram of G6PD from sperm homogenate $(A)$, erythrocyte haemolysate $(B)$, and mixture of sperm homogenate and erythrocyte hemolysate $(A+B)$. The samples were obtained from a normal (G6PD- $B^{+}$individual). The band of haemoglobin $(\mathrm{Hb})$ in haemolysate is indicated in the gel. Extracts were treated with nonionic detergent noniodet-P40 before loading for electrophoresis. The gel electrophoresis and staining were carried out as previously described (Brewer and Dern, 1964).

phoresis of crude extracts, is shown in Fig. 1. Though it is not a standard method of electrophoresis for G6PD, polyacrilamide gel provides a sensitive method for distinguishing the mutant forms of the enzyme molecule in a heterozygote (Howell et al., 1972). The sperm homogenate G6PD activity was inhibited by the neutralizing antibody prepared against the G6PD purified from erythrocyte lysate (Yoshida et al., 1967). The neutralizing antibody was added in the assay mixture a few minutes after the initial rate of G6PD reaction had been established. Addition of antibody in excess, caused immediate inhibition of NADPH formation. In our assay conditions, $5 \mu \mathrm{l}$ antibody neutralized 0.025 units of sperm G6PD activity. Lowered levels of G6PD were found both in sperm and erthyrocytes of an $\mathrm{A}^{-}$ donor (Yoshida et al., 1967) who had only $15 \%$ of normal G6PD activity. Thus it appears that a single genetic locus linked to $\mathrm{X}$ chromosome controls the activity of the 2 isoenzymes. Linder and Gartler 

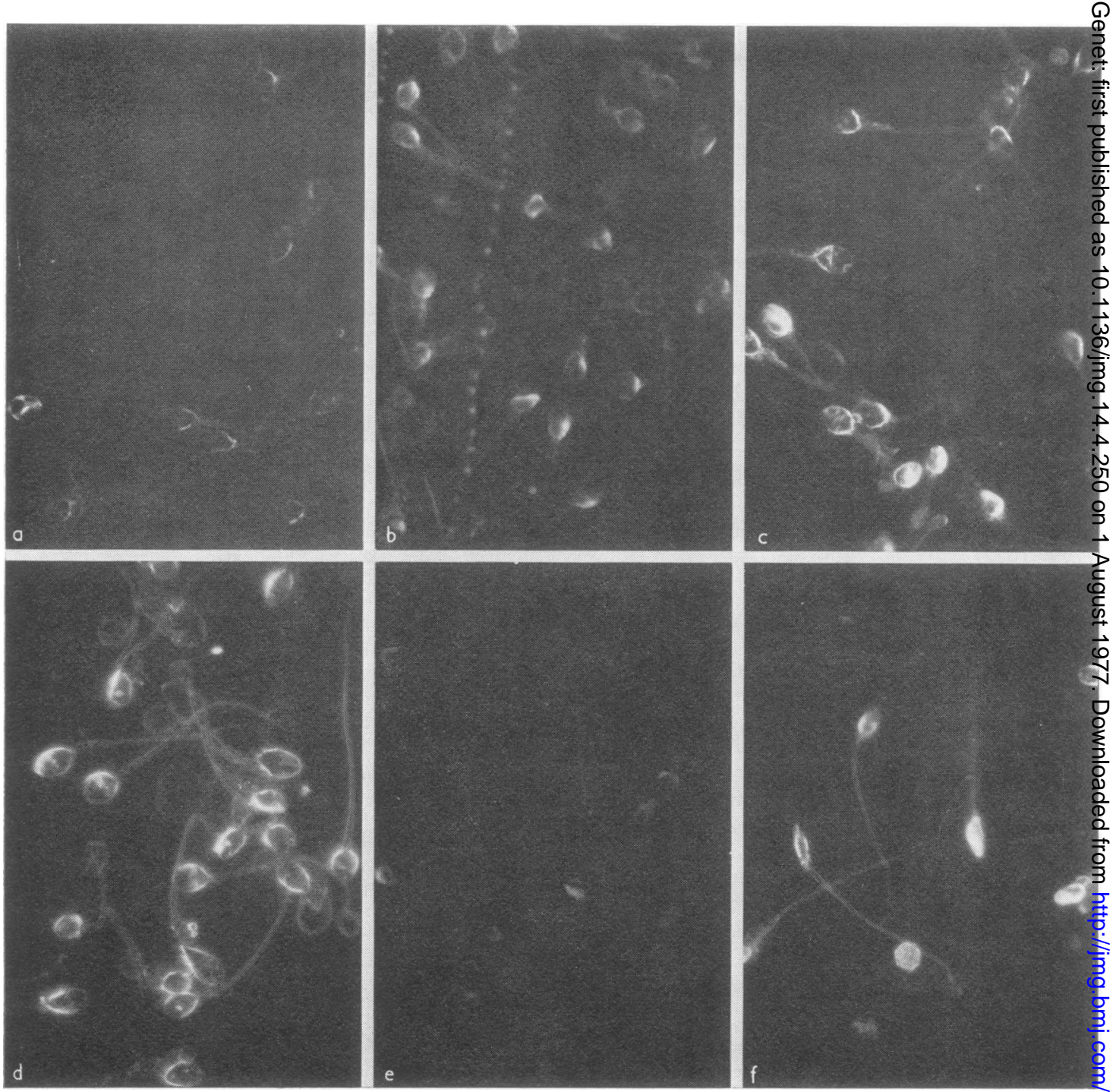

Fig. 2 Fluorescence photomicrograph of human sperm cells incubated to show specific dehydrogenase activities in individual cells. Human sperm incubated (a) with glucose-6-phosphate alone without coenzyme, at room temperature for $D$ 2.5 hours; (b) with $70 \mu \mathrm{M} \mathrm{NADP,} \mathrm{coenzyme} \mathrm{aione,} \mathrm{no} \mathrm{substrate,} \mathrm{at} \mathrm{room} \mathrm{temperature,} 2.5$ hours; (c) in assay mixture for G6PD activity, room temperature, 30 minutes; $(d)$ in assay mixture for 6PGD activity, 15 minutes; $(e) A^{-}$sperm in assay mixture for G6PD activity, $2 \cdot 5$ hours; $(f) A^{-}$sperm in assay mixture for 6PGD activity, 5 minutes.

(1965), from their study of different tissues in females, arrived at the same conclusion.

The specific fluorescence of NADPH produced by individual sperm cells in the presence of G6P and NADP could be visualized under fluorescence microscopy. Thus, we had the opportunity of observing the population distribution of sperm cells with respect to the expression of an X-linked marker. In the early phase of this work, we noticed that washed sperm cells were permeable to phosphorylated్ intermediates, so that we could measure the activities of 2 other X-linked enzymes, phosphoglycerats kinase (PGK) and hypoxanthine-guanine phosphor bosyl transferase (HGPRT) in sperm. However, these? 2 and alphagalactosidase activities could not beo adapted for microscopical observation and G6PБ

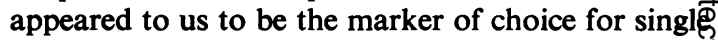
cell studies. Fluorescence microscopy of sperm i 
subjected to many optical artefacts: for example, human sperm has an anisometric head structure, and since the fluorescence is primarily in the head region, approximately half the number of cells which lie on their side may appear nonfluorescent; relatively low activity of G6PD and the fact that G6PD may be inactivated in sperm because of weeks of delay in the passage of sperm from testis to the ejaculate may also explain why a subpopulation of sperm is nonfluorescent. Some of the possible artefacts of observation perhaps could be eliminated if we examined the activity of an NADP dependent dehydrogenase activity which is autosomally linked, since the autosomes segregated symmetrically during male meiosis and one should find such an enzyme to be uniformly active in all cells. The enzyme, 6-phosphogluconate dehydrogenase (6PGD) was chosen for this purpose (McKusick, 1975b); 6PGD is physiologically coupled to the activity of G6PD and also forms reduced NADPH.

The specific fluorescence of NADPH in sperm incubated with NADP and glucose-6-phosphate (G6P) or 6-phosphogluconate (6PG) is shown in Fig. 2. Incubation of sperm after a long period of time ( 2.5 hours) with G6P in the absence of NADP, indicated very low levels of specific fluorescence (Fig. 2a) which may be the result of glucose generated by the hydrolysis of substrate; the background fluorescence is slightly higher after 2.5 hours with NADP alone (Fig. 2b). However, incubation with substrate and coenzyme results in rapid development of fluorescence: sperm incubated with G6P and NADP show brilliant fluorescence in 30 minutes (Fig. 2c), and sperm become fluorescent within 15 minutes when 6PG and NADP are added (Fig. 2d). Deficiency of G6PD in $\mathrm{A}^{-}$sperm produces low fluorescence only after 2.5 hours of incubation (Fig. 2e). However, $\mathrm{A}^{-}$sperm become quickly fluorescent (in 5 minutes) when 6PG and NADP are added (Fig. 2f). The relative amount of fluorescence under the assay conditions was dependent on the period of incubation, as judged by visualization of cells at equal intervals over a period of time, and serially recording fluorescence at constant photographic conditions. Longer periods of incubation were avoided because of an increase in background fluorescence by NADPH escaping into the medium. Independent fluorimetric studies on whole cells suggest that the brightest cells were probably generating in the order of 0.001 picomole of NADPH per minute, and the dark cells or the A-sperm may have no more than $25 \%$ of that activity.

The heterogeniety among normal sperm in the intensity of fluorescence was particularly apparent in the time-lapse photography (Fig. $2 \mathrm{c}$ is a frame from such a series) of cells incubated with G6PD and
Table Relative activities of G6PD and 6PGD in sperm and erythrocytes from normal and $A^{-}$donor*

\begin{tabular}{lcl}
\hline Type of donor & Sperm & Erythrocyte \\
\hline Normal donor: & & \\
G6PD. & 1.0 & 5.21 \\
6PGD. & 2.5 & 4.13 \\
A- donor & & \\
G6PD. & 0.16 & 0.75 \\
6PGD. & 10.05 & 5.03 \\
\hline
\end{tabular}

*The rate of reaction of normal sperm G6PD is taken as unity $(0.001$ pico-mole NADPH produced per minute per sperm) to express the relative activities of both G6PD and 6PGD. Both activities were measured on the same sample by continuous recording of fluorescence generated by sequential addition of substrates. The sequence of addition was reversed to derive values to correct the slopes for each activity, since the product of G6PD reaction is a substrate for 6PGD reaction.

NADP. Sperm from 2 normal donors were scored for the proportion of fluorescent cells under conditions of assaying either for G6PD or 6PGD activity. Cell fluorescence from 6PG and NADP develops very fast, so that counting was done on prints from photographs taken of slides (Fig. 2d). Though the fluorescence appears uniform and present usually in all (above $80 \%$ ) cells, high activity of sperm 6PGD may mask differences that may exist between cells. However, the heterogeneity in the amount of fluorescence was apparent in cells incubated with G6P and NADP (Fig. 2c). In this case, fluorescent sperm was scored directly under microscope; of the 466 cells scored from a given donor (on 4 different ocassions), the results had a range between 36 to $58 \%$. Sperm from a second donor was scored $41 \%$ positive in a sample of 200 cells. Thus, the difference in the population distribution of sperm fluorescence caused by 6PGD and G6PD activities is consistent with what would be expected of the phenotypic expression of autosomal and $\mathrm{X}$-linked markers according to the pattern of chromosomal segregation in male meiosis. Though optical artefacts related to anisometry of the sperm head could be ruled out from these assays, the results do not exclude factors unrelated to genetic segregation, for example, any intrinsic difference in the stability of sperm G6PD and 6PGD which may give rise to heterogeneity in fluorescent sperm distribution.

It appeared to us while examining sperm for 6PGD activity under fluorescence microscopy, that the cells become more rapidly fluorescent, indicating relatively higher activity of 6PGD compared to G6PD. The average ratios of 6PGD:G6PD activities in sperm and erythrocyte homogenates are shown in the Table. The rate of reaction of normal sperm G6PD is taken as unity to express the relative slopes of other activities. The ratio in sperm is approximately twice higher than that in erythrocyte; a higher absolute level of 6PGD in sperm is consistent with the more rapid development of NADPH fluorescence in 
sperm incubated with 6-phosphogluconate than those with glucose-6-phosphate. The activity of 6PGD in $\mathrm{A}^{-}$sperm is even higher, which was not in accordance with the 6PGD levels that have been reported in G6PD deficient individuals (Brewer and Dern, 1964; Parr and Fitch, 1967). It would be interesting to investigate whether all $\mathrm{A}^{-}$donors have extraordinarily high levels of 6PGD in their sperm; such high 6PGD levels suggest that the reduced coenzyme levels in sperm (Mann, 1964) are perhaps controlled by a pathway different from what is known in erythrocyte.

Although many lines of evidence suggest that the condensed XY bivalent is genetically inactive during male meiosis (Monesi, 1971; Moore, 1972) (even deficiencies caused by autosomal mutations or deletions are not limiting for sperm maturation (Hancock, 1972), the possibility of haploid gene expression for an X-linked enzyme cannot be excluded on this basis. Thus we attempted to test the possibility of haploid gene activity for X-linked locus. For example, one would expect that those sperm with low levels of G6PD activity should have $\mathrm{Y}$ and not the $\mathrm{X}$ chromosome. We incubated unfixed sperm preparations with added quinacrine in the reaction mixture for G6PD activity. Staining with quinacrine alone shows the $\mathrm{Y}$ chromosome in 30 to

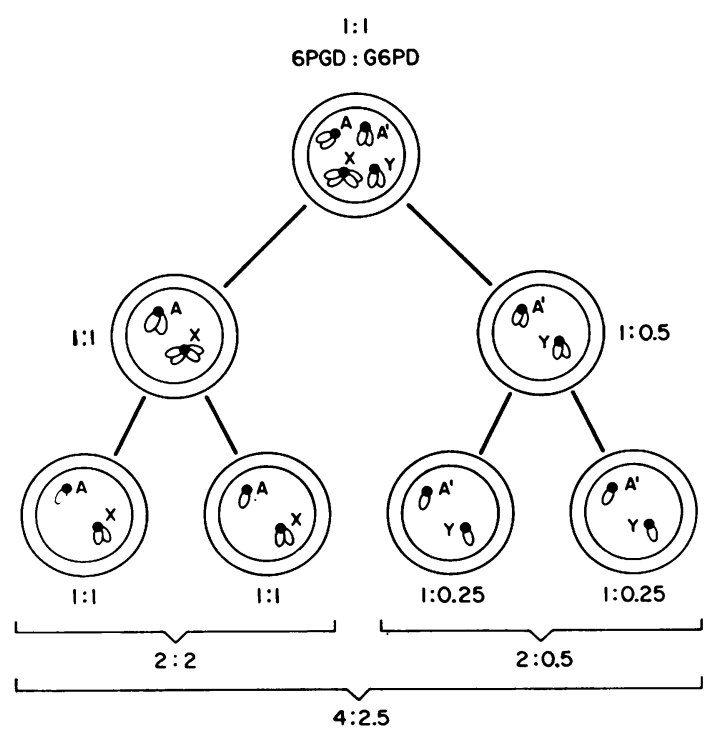

Fig. 3 A schematic model to explain the proportions of 6PGD:G6PD activity to be found in cells after chromosome segregation in spermatogenesis. Autosomes are indicated by the letter $A$ and sex chromsomes by $X$ and $Y$. The principal assumption is that enzyme synthesis is dependent on the presence of chromosomes which remain active during postmeiotic development.
$43 \%$ of cells, a value slightly lower than that of fixe男 cells. Results from the doubly stained cells were्e inconclusive because of technical constraints. The emission spectra of quinacrine and NADPH have considerable overlap which could not be separated by the choice of optical filters. Similarly, the chromosome could not be identified in sperm so thate the high G6PD activity could not be correlated with its presence. The relative rates of 2 activities shown in the Table are however consistent with enzymes synthesis during haploid phase of sperm maturation $\vec{\bullet}$ The expected ratios on each meiotic step, on the basis of relative levels in erythrocyte, are shown irw Fig. 3. In haploid segregants of $Y$ chromosomes? there will be dilution of pre-existing G6PD activit since it can no longer be made in the absence of $X$ chromosome, thus the ratio of 6PGD:G6PD should be 2:0.5; in contrast, in the $X$ chromosomed segregants the ratio should be equal to 1 or less than 1 (range 0.7 to 1.0 ) as it is found in the normas erythrocyte (Linder and Gartler, 1965; Brewer and Dern, 1964; Parr and Fitch, 1967). The overalP expected ratio of 2 enzyme activities as shown is Fig. 3 is compatible with the observed activities? (Table).

Evidence for gene specific phenotypic variations in individual sperm, as we have presented here foj sperm G6PD, will not alone be sufficient to establislo whether genetic expression takes place in haploio spermatids. Our interest in studying $\mathrm{X}$-linke enzyme activities in sperm was because these enzymes may provide an alternative selective procedure for or against a subpopulation of $\operatorname{spern} \vec{B}$ compared with the antigen-antibody system applie $\bar{\Phi}$ by previous workers (Bennett and Boyse, 1973) For example, incubating sperm with guanine. analogues or alternatively deoxyglucose-6-phosphate as a substrate analogue instead of glucose-6-phose phosphate, may preferentially inactivate the class of sperm with high levels of HGPRT or G6PD activity. Treated sperm could then be used in artificial in semination to test for distortion of sex ratio in the progeny. Mouse sperm which probably would be the ideal material for such experiments gave seriouss technical problems in fluorescence microscopy ant. was unsuitable for this type of study; however, both HGPRT and G6PD activities are likely to be sexlinked in mice, and mouse sperm can be used to derive progeny from sperm population preselected on the basis of presence or absence of a specific sex linked enzyme activity.

This work was initiated at The Salk Institute with grants from the Rockefeller Foundation and the Ford Foundation (S.S.) and was later supported b? grant no. GM17702 from the National Institutes op 
Health to the University of California, San Diego (O.W.J.).

\section{References}

Bennett, D., and Boyse, E. A. (1973). Sex ratio in progeny of mice inseminated with sperm treated with $\mathrm{H}-\mathrm{Y}$ antiserum. Nature, 246, 308-309.

Brewer, G. J., and Dern, R. J. (1964). A new inherited enzymatic deficiency of human erythrocytes: 6-phosphogluconate dehydrogenase deficiency. American Journal of Human Genetics. 16, 472-476.

Estabrook, R. W., and Maitra, P. K. (1962). A fluorimetric method for the quantitative microanalysis of adenine and pyridine nucleotides. Analytical Biochemistry, 3, 369-382.

Hancock, J. L. (1972). Spermatogenesis and sperm defects. In Edinburgh Symposium on the Genetics of the Spermatozoon, pp. 121-129. Ed. by R. A. Beatty and S. GluecksohnWaelsch. University of Edinburgh.

Howell, E. B., Nelson, A. J., and Jones, O. W. (1972). A new G6PD variant associated with chronic non-spherocytic hemolytic anaemia in a Negro family. Journal of Medical Genetics, 9, 160-164.

Linder, D., and Gartler, S. M. (1965). Distribution of glucose6-phosphate dehydrogenase electrophoretic variants in different tissues of heterozygotes. American Journal of Human Genetics, 17, 212-220.

McKusick, V. A. (1975a). Mendelian Inheritance in Man, 4th ed., p. 1xv. The John Hopkins University Press, Baltimore, Maryland
McKusick, V. A. (1975b). Mendelian Inheritance in Man, 4th ed., p. 261 (index no. 17220). The Johns Hopkins University Press, Baltimore, Maryland.

Mann, T. (1964). In The Biochemistry of Semen and of the Male Reproductive Tract, pp. 265-307. Barnes and Nobles. Monesi, V. (1971). Chromosome activities during meiosis and spermiogenesis. Journal of Reproduction and Fertility, Suppl. 13. 1-14.

Moore, G. P. M. (1972). A cytological demonstration of the DNA-transcription enzyme RNA polymerase during mammalian spermatogenesis. In Edinburgh Symposium on the Genetics of the Spermatozoon, p.p 90-96. Ed. by R. A. Beatty and S. Gluecksohn-Waelsch. University of Edinburgh.

Parr, C. W., and Fitch, L. I. (1967). Inherited quantitative variations of human phosphogluconate dehydrogenase. Annals of Human Genetics, 30, 339-353.

Yoshida, A. (1966). Glucose 6-phosphate dehydrogenase of human erythrocytes. Journal of Biological Chemistry, 241, 4966-4976.

Yoshida, A., Stamatoyannopoulos, G., and Motulsky, A. G. (1967). Negro variant of glucose-6-phosphate dehydrogenase deficiency $\left(\mathrm{A}^{-}\right)$in man. Science, 155, 97-99.

Requests for reprints to Dr Siddhartha Sarkar, Division of Medical Genetics, Department of Medicine M-013, School of Medicine, University of California, San Diego, La Jolla, California 92093, U.S.A. 